\title{
EFFECT OF LIQUID NITROGEN TREATMENT ON LEAD-FREE (CH3NH3)3BI219 PEROVSKITES FILMS
}

\author{
${ }^{1}$ Anastasiia SOKOLOVA, ${ }^{1}$ Sergei CHEREVKOV, ${ }^{1}$ Ivan SKURLOV, ${ }^{1}$ Aleksandr LITVIN, \\ ${ }^{1,2}$ Elena USHAKOVA, ${ }^{1}$ Anatoly FEDOROV, ${ }^{1}$ Alexander BARANOV \\ 1/TMO University, Center of Information Optical Technologies, Saint Petersburg, Russian Federation, \\ avsokolova@itmo.ru \\ ${ }^{2}$ City University of Hong Kong, Department of Materials Science and Engineering, and Center for Functional \\ Photonics (CFP), Hong Kong S.A.R.
}

https://doi.org/10.37904/nanocon.2019.8551

\begin{abstract}
Metal and organometal halide perovskites, an emerging class of direct bandgap semiconductors, have attracted great attention lately. Lead halide perovskites were recognized as promising candidates for future lighting applications, due to their high quantum yield, narrow full width at half maximum (FWHM), and wide color gamut. Nonetheless, the toxicity of lead induces new approaches such as obtaining lead-free perovskites and further investigation of their stability and optoelectronic properties.

In this work is presented a study of lead-free perovskites $(\mathrm{CH} 3 \mathrm{NH} 3) 3 \mathrm{Bi} 219$ film formation for a further application in optoelectronic devices. The experimental part consists of the formation $(\mathrm{CH} 3 \mathrm{NH} 3) 3 \mathrm{Bi} 219$ films and a treatment part. First part has been performed with a quite popular spin-coating approach that provides accessibility and simplicity of the sample's preparation. Secondly, a liquid nitrogen treatment has been carried out, afterward was investigated the effect of this treatment on the lead-free perovskites films.
\end{abstract}

Keywords: Perovskite, films, lead-free, emission, stability

\section{INTRODUCTION}

Lead-free perovskites are a promising class of eco-friendly materials for applications in almost all spheres of nowadays photonics of nanostructures. As their more investigated lead analogues, lead-free perovskites are attracted huge interest of scientists due to their potentially high quantum yield, narrow full width at half maximum (FWHM), and wide color gamut.

The most popular candidates to replacing lead are tin(II) (ABX3 structure)[1] , tin(IV) in double perovskites [2], bismuth(III) [3] and antimony(III) [4] in triple (A3B2X9) and double hybrid perovskites (A2B'B"X6) [5]. All candidates from this list are theoretically shown as elements that maintain the stability in the crystalline lattice similarly to lead halide perovskites (LHP). However, on practice even the task of obtaining lead-free perovskites is non-trivial. The most usable method for synthesis lead-free perovskites for future applications and investigations of their special optical properties is a making films with a wide range of approaches that is already popular in production of photovoltaic devices. This way of obtaining lead-free perovskites gives a flexibility during preparation of samples and do not request special equipment such as Schlenk lines or some depositional machines. In this work only spin-coater and desiccator with inert atmosphere were used. Glovebox isn't required for an experiment first of all because of the film's composition: Bi gives an oxidation stability in comparing with other substitution candidates that mentioned above. Furthermore, for increasing the surface morphology and optical properties of perovskites films were investigated new non-chemical approach as liquid nitrogen treatment that provides cheap, accessible and easy technology. 


\section{EXPERIMENTAL}

Bismuth iodide (Bil3, 99\%), anhydrous 2-propanol (iPrOH, 99.5\%) and N,N-Dimethylformamide (DMF, $\geq 99,9 \%$ ) were purchased from Sigma-Aldrich, methylammonium iodide (MAI, 99\%) was purchased from J\&K. The chemicals were used without any additional purification.

All films were deposited onto glass substrates that was prepared by washing with soap and solvents such as $\mathrm{CHCl} 3$, toluene, acetone, iPrOH with further plasma-cleaner treatment.

Films were synthesized by two different techniques. First protocol was published by Hoye [6]. Briefly, $80 \mathrm{mg}$ Bil3 was dissolved into $0.2 \mathrm{ml}$ of DMF with following ultrasonication for 30 minutes. After the solution was filtered with $0.22 \mu \mathrm{m}$ syringe filter and spin-coated onto glass substrate on $3000 \mathrm{rpm}$ for $5 \mathrm{~s}$ and $6000 \mathrm{rpm}$ for another $5 \mathrm{~s}$ after. That layer was dried in desiccator on $70{ }^{\circ} \mathrm{C}$ for 30 minutes. Layer had a dark red metallic color. At that time another precursor was prepared by dissolving $6 \mathrm{mg}$ of MAl in $1 \mathrm{~mL}$ of anhydrous $\mathrm{iPrOH}$. Second layer of MAI solution was deposited with similar approach but with pause for $40 \mathrm{~s}$ before spin-coating and different speed and time $-4000 \mathrm{rpm}$ for $30 \mathrm{~s}$. After dripping of MAI source film was changed color from described above to bright orange. Further annealing step on $100^{\circ} \mathrm{C}$ for $1 \mathrm{~h}$ in inert atmosphere was performed.

Second protocol was published by Senol Öz [7]. The precursor solution was prepared by first dissolving $69 \mathrm{mg}$ MAl in $1 \mathrm{ml}$ DMF followed by addition of $168 \mathrm{mg}$ Bil3 to obtain a clear red $20 \mathrm{wt} \%$ solution. The precursor solution was heated to $60{ }^{\circ} \mathrm{C}$ and spin-coated subsequently at $4000 \mathrm{rpm}$ for 45 seconds. The films were annealed at $100^{\circ} \mathrm{C}$ for $30 \mathrm{~min}$ in an inert atmosphere.

Steady-state spectral measurements of samples were carried out using a UV-3600 spectrophotometer (Shimadzu) Atomic force microscopy (AFM) measurements were taken with Solver-Pro (NT-MDT, Russia) atomic force microscope in the semicontact mode.

\section{RESULTS AND DISCUSSION}
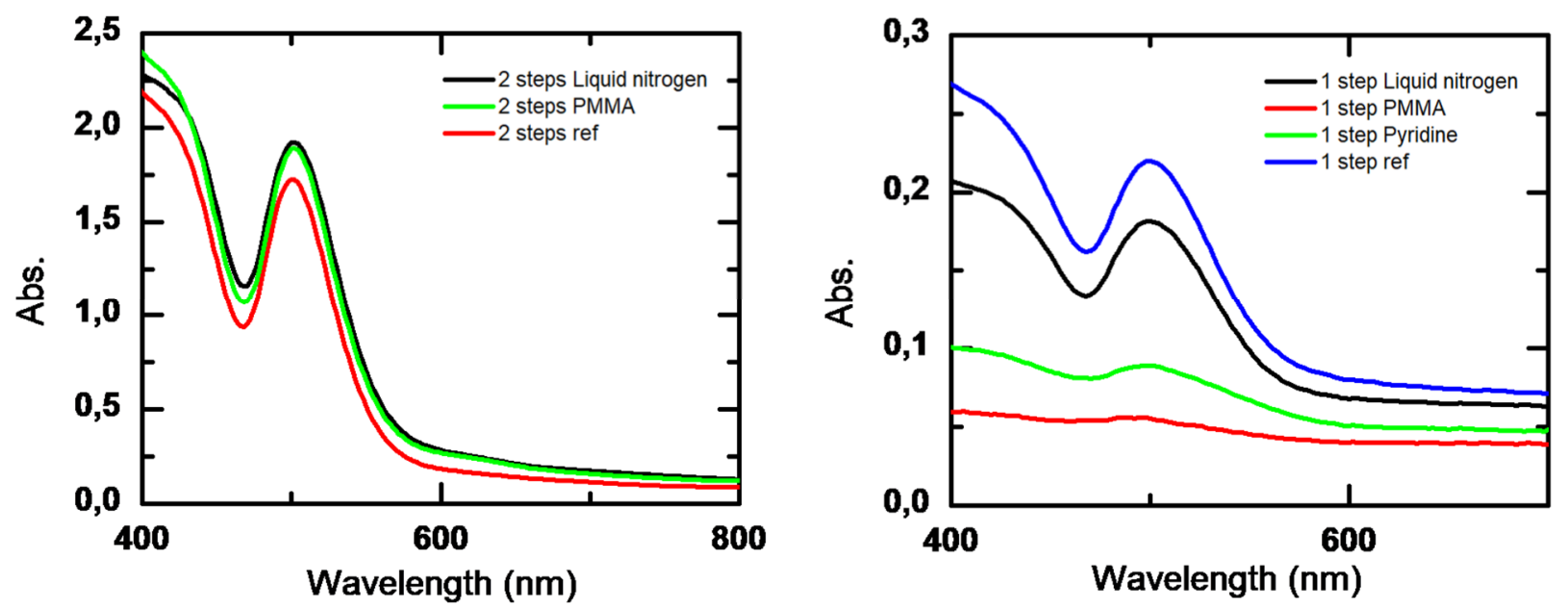

Figure 1 Absorption spectra of films that were produced by two different approaches: 1 and 2 step synthesis, respectively

The Figure 1 shows the absorption spectra of films synthesis of which was described above. Two steps films show better quality of crystal structure that could be concluded from the peak shape: peak is narrow and with explicit borderline. Peak positions are similar between two methods that is an evidence of happened crystallization processes during production of films. However, with both films oxidation and light influence is critical and lead to faster degradation. During experiment two different approaches to liquid nitrogen treatment 
were used. Firstly, $\mathrm{N}_{2}$ was dropped just on the plate with substrate on air without any other special actions. As it might me noticed, such treatment extinguished even abs, color of film has changed from bright yellow to yellowish. This degradation has explanation in degradation of surface due to hydration from condensed from air water-gas. Such factors as high humidity play key roles even during deposition process and become borders to simplifying and cheapening the manufacturing procedures. There're still some questions of overcoming such problems without using gloveboxes or other special equipment that are interesting aims for our future work.
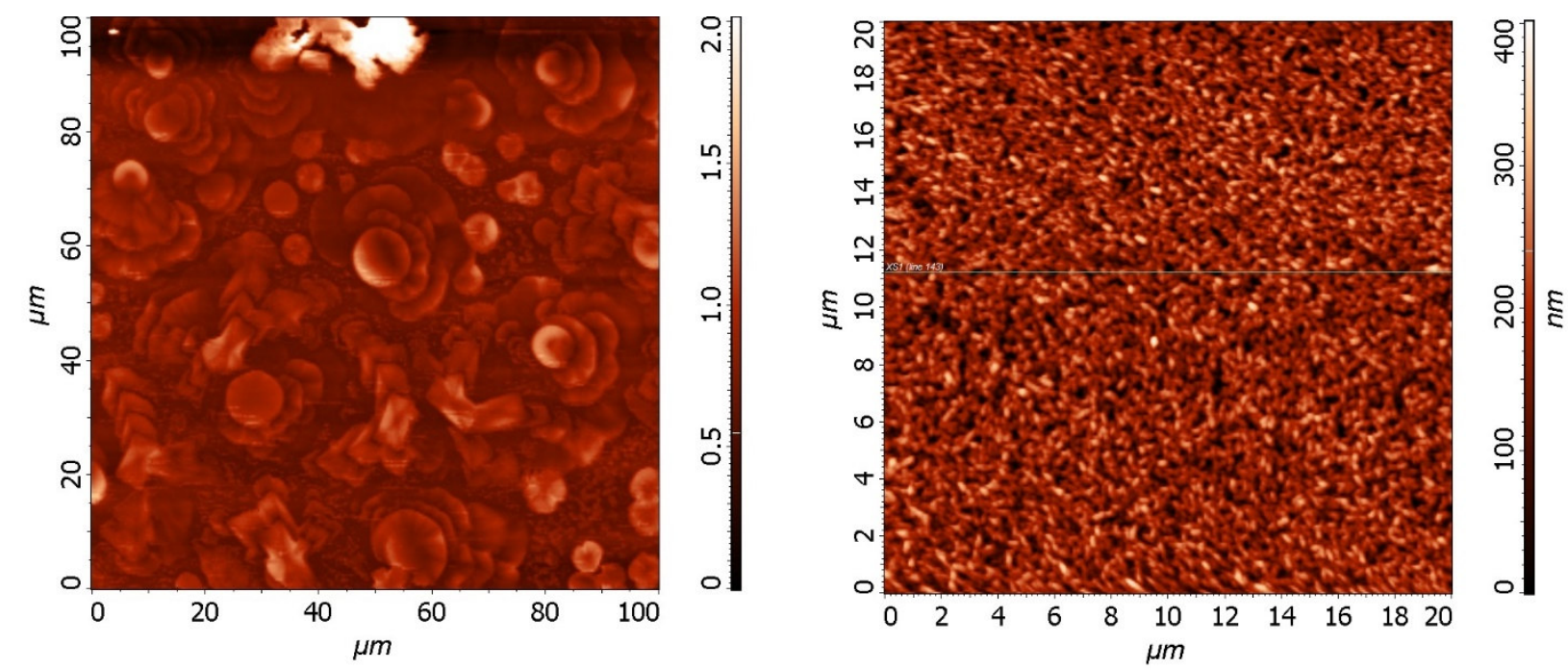

Figure 2 The AFM images of the surface of film before and after liquid nitrogen treatment.

Film was made by 2 steps synthesis procedure.

By next step, because of no significant changes in absorption spectra were observed, AFM investigation of surface has taken place. During this measurement have been watched noticeable differences between films with and without nitrogen liquid treatment that could be seen in Figure 2. The smaller grain size and better morphology is obvious from these images. Interesting that similar changes are taken place during chemical types of treatment such as pyridine or PMMA $[8,9]$. Assumed, that extremely low temperature lead to increasing of stability in high levels of crystalline lattice that is observed and described above. The behavior of films and theories of this behavior is still argued that are required paying more attention.

\section{CONCLUSION}

In conclusion, we introduce a method for a non-trivial and simple treatment of perovskite films that save the optical density of film during procedure i.e. not decreasing the quantity of layers but the same time lead to improvements in the surface quality. Such easy accomplished procedure might be used in manufacturing of future perovskites applications without their rise in price.

\section{ACKNOWLEDGEMENTS}

This work was supported by the Russian Science Foundation (Agreement 18-13-00200).

\section{REFERENCES}

[1] Synthesis and Optical Properties of Lead-Free Cesium Tin Halide Perovskite Nanocrystals. Tom C. Jellicoe, Johannes M. Richter, Hugh F. J. Glass, Maxim Tabachnyk et al. Journal of the American Chemical Society 2016138 (9), 2941-2944 https://doi.org/10.1021/jacs.5b13470 
[2] Controlled Synthesis of Lead-Free and Stable Perovskite Derivative Cs2Snl6 Nanocrystals via Facile HotInjection Process. Aifei Wang, Xingxu Yan, Mian Zhang, Shibin Sun et al. Chemistry of Materials 201628 (22), 8132-8140. https://doi.org/10.1021/acs.chemmater.6b01329

[3] Synthesis and Optical Properties of Colloidal M3Bi2l9 (M = Cs, Rb) Perovskite Nanocrystals. Jaya Pal, Amit Bhunia, Sudip Chakraborty, Suman Manna et al. The Journal of Physical Chemistry C 2018122 (19), 1064310649 https://doi.org/10.1021/acs.jpcc.8b03542

[4] High Quantum Yield Blue Emission from Lead-Free Inorganic Antimony Halide Perovskite Colloidal Quantum Dots. Jian Zhang, Ying Yang, Hui Deng, Umar Farooq et al. ACS Nano 201711 (9), 9294-9302 https://doi.org/10.1021/acsnano.7b04683

[5] The Making and Breaking of Lead-Free Double Perovskite Nanocrystals of Cesium Silver-Bismuth Halide Compositions. Yehonadav Bekenstein, Jakob C. Dahl, Jianmei Huang, Wojciech T. Osowiecki et al. Nano Letters 201818 (6), 3502-3508 https://doi.org/10.1021/acs.nanolett.8b00560

[6] Hoye, R. L. Z., Brandt, R. E., Osherov, A., Stevanović, V., Stranks, S. D., Wilson, M. W. B., ... Buonassisi, T. (2016). Methylammonium Bismuth lodide as a Lead-Free, Stable Hybrid Organic-Inorganic Solar Absorber. Chemistry - A European Journal, 22(8), 2605-2610. https://doi.org/10.1002/chem.201505055

[7] Öz, S., Hebig, J. C., Jung, E., Singh, T., Lepcha, A., Olthof, S., ... \& Meerholz, K. (2016). Zero-dimensional (CH3NH3) 3Bi2l9 perovskite for optoelectronic applications. Solar Energy Materials and Solar Cells, 158, 195201. https://doi.org/10.1016/j.solmat.2016.01.035Get

[8] de Quilettes, D. W., Vorpahl, S. M., Stranks, S. D., Nagaoka, H., Eperon, G. E., Ziffer, M. E., ... \& Ginger, D. S. (2015). Impact of microstructure on local carrier lifetime in perovskite solar cells. Science, 348(6235), 683-686. https://doi.org/10.1126/science.aaa5333

[9] Bi, D., Yi, C., Luo, J., Décoppet, J. D., Zhang, F., Zakeeruddin, S. M., ... \& Grätzel, M. (2016). Polymer-templated nucleation and crystal growth of perovskite films for solar cells with efficiency greater than $21 \%$. Nature Energy, 1(10), 16142. https://doi.org/10.1038/NENERGY.2016.142. 